\title{
CHARACTERIZATION OF EIGENFUNCTIONS OF THE LAPLACIAN BY BOUNDEDNESS CONDITIONS
}

\author{
ROBERT S. STRICHARTZ
}

\begin{abstract}
If $\left\{f_{k}(x)\right\}_{k \in \mathbb{Z}}$ is a doubly infinite sequence of functions on $\mathbb{R}^{n}$ which are uniformly bounded and such that $\Delta f_{k}=f_{k+1}$, then $\Delta f_{0}=-f_{0}$. This generalizes a theorem of Roe $(n=1)$. The analogous statement is true on the Heisenberg group, but false in hyperbolic space.
\end{abstract}

\section{INTRODUCTION}

A function on the line with the property that all its derivatives and antiderivatives are uniformly bounded must be a linear combination of $\sin x$ and $\cos x$, in other words an eigenfunction of $d^{2} / d x^{2}$ with eigenvalue -1 [R]. Since antiderivatives are not unique, the hypothesis can be stated more precisely as follows: there exists a doubly infinite sequence $\left\{f_{k}(x)\right\}_{k \in \mathbb{Z}}$ of functions on the line such that

$$
\frac{d}{d x} f_{k}=f_{k+1}
$$

and

$$
\left|f_{k}(x)\right| \leq M \quad \text { for all } k \in \mathbb{Z}, \quad x \in \mathbb{R} .
$$

Here we look for an $n$-dimensional generalization. In place of derivatives and antiderivatives we use powers of the Laplacian. Our main result is the following:

Theorem 1.1. Let $\left\{f_{k}(x)\right\}_{k \in \mathbb{Z}}$ be a doubly infinite sequence of functions on $\mathbb{R}^{n}$ satisfying

$$
\begin{gathered}
\Delta f_{k}=f_{k+1}, \\
\left|f_{k}(x)\right| \leq M \quad \text { for all } k \in \mathbb{Z}, \quad x \in \mathbb{R}^{n} .
\end{gathered}
$$

Then $\Delta f_{0}=-f_{0}$.

Of course there are many bounded eigenfunctions of $\Delta$. If $d \mu$ is any finite measure on the unit sphere then $\int e^{i x \cdot \xi} d \mu(\xi)$ is a bounded eigenfunction, but there are other bounded eigenfunctions not of this form. It would appear to be difficult to give a structure theorem for all bounded eigenfunctions.

The proof of Theorem 1.1 is given in $\S 2$, using harmonic analysis. The proof is similar in spirit to the one-dimensional result of Roe [R] (or the proof in

Received by the editors July 12, 1989 and, in revised form, May 30, 1991.

1991 Mathematics Subject Classification. Primary 42B10, 35J05; Secondary 43A80.

Research supported in part by the National Science Foundation.

(C) 1993 American Mathematical Society $0002-9947 / 93 \$ 1.00+\$ .25$ per page 
[Ho]), in that we show first that the Fourier transform of $f_{0}$ is a distribution supported on the unit sphere. Of course Roe's theorem could be easily stated to a calculus class; can anyone find a proof that could be explained to a calculus class?

It might seem plausible to conjecture that Theorem 1.1 could be extended to Laplacians in different contexts. In $\S 3$ we give a few such extensions, but also a simple counterexample for the case of hyperbolic space. This should not be too surprising if we recall that harmonic analysis in $\mathbb{R}^{n}$ has the peculiar property that the $L^{2}$ theory is powerful enough to handle the $L^{\infty}$ theory; in hyperbolic space this is no longer the case. In $\S 4$ we prove the analogue of the main theorem for the Heisenberg group. The argument uses harmonic analysis on the Heisenberg group, but another idea is needed to clinch the proof. This is probably a reflection of the fact that there is a family of one-dimensional representations of the Heisenberg group that do not play a role in the usual $L^{2}$ harmonic analysis.

The proof of the main theorem uses standard facts about harmonic analysis in $\mathbb{R}^{n}$ and distribution theory that can be found in [GS] or [SW].

Related results concerning distributions whose Fourier transforms vanish on specified sets have been obtained by Gabardo [ $\mathrm{Ga} 1, \mathrm{Ga} 2]$. After this paper was completed, some of the results were generalized by Howard and Reese [HR] to allow more general differential operators and to allow polynomial growth in the estimates.

\section{Proof of THE MAIN THEOREM}

We break the proof up into three steps. In the first step we consider the Fourier transform $\hat{f}_{0}$ of $f_{0}$, which exists as a tempered distribution because $f_{0}$ is bounded.

Lemma 2.1. Under the hypotheses of Theorem 1.1, the distribution $\hat{f}_{0}$ is supported on the unit sphere.

Proof. First we show that $\hat{f}_{0}$ is supported in $|x| \leq 1$. To do this we need to show that $\left\langle\hat{f}_{0}, \varphi\right\rangle=0$ for any test function $\varphi$ supported in $|x|>1$. Now observed that (1.3) means

$$
|x|^{2 k} \hat{f}_{0}(x)=(-1)^{k} \hat{f}_{k}(x)
$$

as distributions, for any $k \geq 0$. Therefore

$$
\left\langle\hat{f}_{0}, \varphi\right\rangle=\left\langle|x|^{2 k} \hat{f}_{0},|x|^{-2 k} \varphi\right\rangle=(-1)^{k}\left\langle f_{k}, \mathscr{F}\left(|x|^{-2 k} \varphi\right)\right\rangle ;
$$

hence

$$
\left|\left\langle\hat{f}_{0}, \varphi\right\rangle\right| \leq M\left\|\mathscr{F}\left(|x|^{-2 k} \varphi\right)\right\|_{1}
$$

by (1.4). Now we use the well-known estimate

$$
\|\mathscr{F} g\|_{1} \leq c \sum_{|\alpha| \leq n+1}\left\|\left(\frac{\partial}{\partial x}\right)^{\alpha} g\right\|_{1}
$$

applied to $g=|x|^{-2 k} \varphi$. Since $\varphi$ is supported in $|x| \geq 1+\varepsilon$ for some fixed $\varepsilon>0$, it is clear that $(\partial / \partial x)^{\alpha}\left(|x|^{-2 k} \varphi\right) \rightarrow 0$ in $L^{1}$ norm as $k \rightarrow \infty$ for any 
fixed $\alpha$. Thus the right side of (2.2) tends to zero as $k \rightarrow \infty$, so $\left\langle\hat{f}_{0}, \varphi\right\rangle=0$ as claimed.

To complete the proof we need to show that $\hat{f}_{0}$ is also supported in $|x| \geq 1$, which means $\left\langle\hat{f}_{0}, \varphi\right\rangle=0$ if $\varphi$ is supported in $|x|<1$. Here we use (1.3) to obtain

$$
\left\langle\hat{f}_{0}, \varphi\right\rangle=(-1)^{k}\left\langle\hat{f}_{-k},|x|^{2 k} \varphi\right\rangle
$$

and the argument proceeds as before.

The next step in the proof is a structure theorem for distributions supported on the unit sphere. Since the sphere is a smooth compact manifold, this result is well known and we omit the proof.

Lemma 2.2. A distribution $T$ on $\mathbb{R}^{n}$ supported on the unit sphere $S^{n-1}$ has the form

$$
\sum_{k=0}^{N}\left(x \cdot \frac{\partial}{\partial x}\right)^{k} T_{k}
$$

for some finite $N$, where $T_{k}$ are distributions on $S^{n-1}$. In other words

$$
\langle T, \varphi\rangle=\sum_{k=0}^{N}\left(r \frac{\partial}{\partial r}\right)^{k}\left\langle T_{k}(u), \varphi(r u)\right\rangle_{S^{n-1}},
$$

where $u$ denotes a variable on $S^{n-1}$ and $\langle,\rangle_{S^{n-1}}$ denotes the pairing between distributions and test functions on $S^{n-1}$.

The combination of the two lemmas shows that $\hat{f}_{0}$ has the form (2.4). To complete the proof of the theorem we have to show that $N=0$ in (2.4). To do this we use the identity

$$
|x|^{2 j} \sum_{k=0}^{N}\left(x \cdot \frac{\partial}{\partial x}\right)^{k} T_{k}=\sum_{k=0}^{N}\left(x \cdot \frac{\partial}{\partial x}-2 j\right)^{k} T_{k} .
$$

The proof of (2.6) is an easy exercise based on the computation

$$
|x|^{2 j}\left(x \cdot \frac{\partial}{\partial x}\right)=\left(x \cdot \frac{\partial}{\partial x}-2 j\right)|x|^{2 j}
$$

and the fact that $|x|^{2 j} T_{k}=T_{k}$ since $T_{k}$ is a distribution on $S^{n-1}$.

Now we take the inverse Fourier transform of (2.6) and obtain

$$
f_{j}=\sum_{k=0}^{N}(2 j)^{k} F_{k}
$$

where $F_{k}$ are fixed functions (independent of $j$ ) and $\widehat{F}_{N}= \pm T_{N}$. It is easy to see that the functions $F_{k}$ must be bounded, and since $\left\|(2 j)^{N} F_{N}\right\|_{\infty}=$ $(2 j)^{N}\left\|F_{N}\right\|_{\infty}$ while $\left\|\sum_{k=0}^{N-1}(2 j)^{k} F_{k}\right\|_{\infty}=O\left(j^{N-1}\right)$ as $j \rightarrow \infty$, we conclude that $F_{N} \equiv 0$ if $N>0$. This proves that we can take $N=0$ in (2.4), hence $\Delta f_{0}=-f_{0}$, which completes the proof of the theorem.

\section{EXTENSIONS AND EXAMPLES}

Suppose we replace the Laplacian by a d'Alembertian,

$$
\square=\frac{\partial^{2}}{\partial x_{1}^{2}}+\cdots+\frac{\partial^{2}}{\partial x_{p}^{2}}-\frac{\partial^{2}}{\partial x_{p+1}^{2}}-\cdots-\frac{\partial^{2}}{\partial x_{n}^{2}} \quad \text { for } 1 \leq p \leq n-1 .
$$


Then there exist bounded solutions to the eigenfunctions equations $\square f= \pm f$. We have the following generalization of the main theorem.

Theorem 3.1. Let $\left\{f_{k}(x)\right\}_{x \in \mathbb{Z}}$ be a doubly infinite sequence of functions on $\mathbb{R}^{n}$ satisfying

$$
\begin{gathered}
\square f_{k}=f_{k+1}, \\
\left|f_{k}(x)\right| \leq M \quad \text { for all } x \in \mathbb{Z}, \quad x \in \mathbb{R}^{n} .
\end{gathered}
$$

Then $f_{0}=f_{+}+f_{-}$where $f_{ \pm}$are bounded solutions of $\square f_{ \pm}= \pm f_{ \pm}$.

Proof. The proof follows the same outline as before, with one additional twist. Let $[x, y]=x_{1} y_{1}+\cdots+x_{p} y_{p}-x_{p+1} y_{p+1}-\cdots-x_{n} y_{n}$. Then the proof of Lemma 2.1 extends to show that $\hat{f}_{0}$ is supported on the pair of hyperboloids $[x, x]=$ \pm 1 . However, because these hyperboloids are not compact, the analogue of Lemma 2.2 only holds locally. Thus we need to perform a localization on the Fourier transform side, which conveniently does not alter the hypotheses of the theorem. Let $\psi$ be any compactly supported test function, and let $g_{j}=$ $\mathscr{F}^{-1}\left(\psi \hat{f}_{j}\right)$. Then $\left\{g_{j}\right\}_{j \in \mathbb{Z}}$ satisfies (3.1) and (3.2); in fact the constant in (3.2) is only multiplied by $\left\|\mathscr{F}^{-1} \psi\right\|_{1}$, which we can easily arrange to be equal to one.

Now $\hat{g}_{0}$ is compactly supported on the union of hyperboloids which are smooth manifolds, and $x \cdot \partial / \partial x$ is a transverse directional derivative. Therefore, by the analogue of Lemma 2.2, we have

$$
\hat{g}_{0}=\sum_{k=0}^{N}\left(x \cdot \frac{\partial}{\partial x}\right)^{k}\left(T_{k}^{+}+T_{k}^{-}\right)
$$

where $T_{k}^{ \pm}$are distributions on $[x, x]= \pm 1$. We can then show that $N=0$ as before because we have

$$
[x, x]^{j} \sum_{k=0}^{N}\left(x \cdot \frac{\partial}{\partial x}\right)^{k} T_{k}^{ \pm}=( \pm 1)^{j} \sum_{k=0}^{N}\left(x \cdot \frac{\partial}{\partial x}-2 j\right)^{k} T_{k}^{ \pm}
$$

as the analogue of (2.6).

Now from (3.3) with $N=0$ we have $g_{0}=g_{+}+g_{-}$where $\square g_{ \pm}= \pm g_{ \pm}$. Note that then $g_{1}=\square g_{0}=g_{+}-g_{-}$so that $g_{ \pm}$are both bounded functions with the same bound $M$ as appeared in (3.2). Then a routine limiting argument with $\psi \rightarrow 1$ yields the same conclusion for $f_{0}$.

Another straightforward extension of the main theorem is to replace $L^{\infty}$ norms by $L^{p}$ norms; in other words, to replace (1.4) by the condition

$$
\left\|f_{k}\right\|_{p} \leq M \text { for all } k \in \mathbb{Z} \text {. }
$$

Of course for the result to be nonvacuous we need to take $p>2 n /(n-1)$; in particular there is nothing new when $n=1$.

The harmonic oscillator operator $-\Delta+|x|^{2}$ provides another generalization of the main theorem in which we need only consider positive powers of the operator.

Theorem 3.2. Let $f$ be a function on $\mathbb{R}^{n}$ satisfying

$$
\left\|\left(-\Delta+|x|^{2}\right)^{k} f\right\|_{\infty} \leq M n^{k} \quad \text { for all } k \geq 0 .
$$

Then $f(x)=c e^{-|x|^{2} / 2}$. 
Proof. Let $H_{\alpha}(x)$ denote the Hermite functions of order $\alpha=\left(\alpha_{1}, \ldots, \alpha_{n}\right)$, which form a basis of eigenfunctions for the harmonic oscillator with

$$
\left(-\Delta+|x|^{2}\right) H_{\alpha}(x)=(n+2|\alpha|) H_{\alpha}(x) .
$$

Then

$$
\left\langle\left(-\Delta+|x|^{2}\right)^{k} f, H_{\alpha}\right\rangle=\left\langle f,\left(-\Delta+|x|^{2}\right)^{k} H_{\alpha}\right\rangle=(n+2|\alpha|)^{k}\left\langle f, H_{\alpha}\right\rangle
$$

while (3.6) implies

$$
\left|\left\langle\left(-\Delta+|x|^{2}\right)^{k} f, H_{\alpha}\right\rangle\right| \leq M n^{k}\left\|H_{\alpha}\right\|_{1} .
$$

Thus $\left\langle f, H_{\alpha}\right\rangle=0$ for all $\alpha \neq 0$, and so by choosing the constant $c$ appropriately we obtain $\left\langle f-c e^{-|x|^{2} / 2}, H_{\alpha}\right\rangle=0$ for all $\alpha$. To complete the proof we need to invoke the uniqueness of Hermite expansions for $L^{\infty}$ functions. In fact, if $T$ is any tempered distribution then $\left\langle T, H_{\alpha}\right\rangle=0$ for all $\alpha$ implies $T=0$ (see [RS] for the essential ideas of a proof).

Next we consider a negative example. Let $\Delta$ denote the Laplace-Beltrami operator on hyperbolic 3-space $H^{3}$. The computations in this case are especially transparent, but the same phenomenon occurs for hyperbolic space of any dimension, or more generally for noncompact symmetric spaces (see [He]).

Choose an origin in hyperbolic space, and let $r$ denote the hyperbolic distance to the origin. Then $(\operatorname{see}[\mathrm{He}])$ the functions $(\sin \lambda r) /(\sinh r)$ for $\lambda$ real are the spherical functions on $H^{3}$ and satisfy the eigenfunction equation

$$
\Delta\left(\frac{\sin \lambda r}{\sinh r}\right)=-\left(1+\lambda^{2}\right)\left(\frac{\sin \lambda r}{\sinh r}\right) .
$$

However, it is not necessary to restrict $\lambda$ to be real; indeed (3.8) is an analytic identity in $\lambda$ so it is valid for all $\lambda \in \mathbb{C}$. It is obvious by inspection that the function $(\sin \lambda r) /(\sinh r)$ is bounded if and only if $|\operatorname{Im} \lambda| \leq 1$. On the other hand we can make the eigenvalue $-1-\lambda^{2}$ have absolute value equal to one by taking

$$
\lambda=i\left(1+e^{i \theta}\right)^{1 / 2},
$$

and it is clear that for $\theta$ close to $\pi$ we will have $|\operatorname{Im} \lambda| \leq 1$. It is clear that $f_{k}=e^{i k \theta}(\sin \lambda r) /(\sinh r)$ with $\lambda$ given by (3.9) gives a sequence satisfying the analogues of (1.3) and (1.4), so the theorem does not extend to this context.

\section{The HeISENBerg group}

The Heisenberg group Heis $n$ is the space $\mathbb{C}^{n} \times \mathbb{R}$ equipped with the group law

$$
(z, t) \circ(w, s)=\left(z+w, t+s+\frac{1}{2} \operatorname{Im} z \cdot \bar{w}\right) .
$$

The vector fields

$$
\begin{aligned}
& T=\frac{\partial}{\partial t}, \\
& L_{j}=\frac{\partial}{\partial x_{j}}-\frac{1}{2} y_{j} \frac{\partial}{\partial t}, \quad j=1, \ldots, n, \\
& M_{j}=\frac{\partial}{\partial y_{j}}+\frac{1}{2} x_{j} \frac{\partial}{\partial t}, \quad j=1, \ldots, n,
\end{aligned}
$$


form a basis for the Lie algebra of left-invariant vector fields, and the operator

$$
\mathscr{L}=\sum_{j=1}^{n}\left(L_{j}^{2}+M_{j}^{2}\right)
$$

is called the Heisenberg Laplacian. It is not difficult to see that there are many bounded eigenfunctions satisfying $\mathscr{L} f=-f$, for example $f(z, t)=$ $e^{ \pm i t / n} e^{-|z|^{2} / 4 n}([\operatorname{Str} 1, \S 6]$, or [Ge 1]).

Theorem 4.1. Let $\left\{f_{k}\right\}_{k \in \mathbb{Z}}$ be a doubly infinite sequence of functions on Heis ${ }_{n}$ satisfying

$$
\begin{gathered}
\mathscr{L} f_{k}=f_{k+1} \\
\left\|f_{k}\right\|_{\infty} \leq M \quad \text { for all } k \in \mathbb{Z} .
\end{gathered}
$$

Then $\mathscr{L} f_{0}=-f_{0}$.

Proof. The proof is based on the harmonic analysis of $\mathrm{Heis}_{n}$. We recall from [Str 1] that there exist functions $\varphi_{\alpha, \beta, \varepsilon}(z)$ on $\mathbb{C}^{n}$ where $\alpha, \beta$ are $n$-multiindices and $\varepsilon= \pm 1$ which are polynomials in $z$ times $e^{-|z|^{2} / 2}$ (in [Str 1] the polynomials are given by certain special Hermite polynomials, but [Fo] shows that they may also be expressed in terms of Laguerre polynomials, which is the form given in [Ge 1]. See also [Str 2].) For each fixed $\varepsilon= \pm 1$, the functions $\varphi_{\alpha, \beta, \varepsilon}$ form an orthonormal basis for $L^{2}\left(\mathbb{C}^{n}\right)$, and what is significant for the harmonic analysis on $\mathrm{Heis}_{n}$ is that the function

$$
h_{\rho}(z, t)=e^{i \varepsilon \rho t /(n+2|\alpha|)} \varphi_{\alpha, \beta, \varepsilon}\left(\frac{\sqrt{\rho} z}{\sqrt{4|\alpha|+2 n}}\right)
$$

satisfies

$$
\mathscr{L} h_{\rho}(z, t)=-\rho h_{\rho}(z, t) .
$$

Note that the functions $h_{\rho}$ do not decay in the $t$-direction, but if we take a smooth average with respect to $\rho$ we can obtain any desired decay. Suppose $\psi(\rho)$ is a test function with compact support in $(0, \infty)$. Then an integration by parts argument shows that $\int_{0}^{\infty} h_{\rho}(z, t) \psi(\rho) d \rho$ is integrable on $\mathrm{Heis}_{n}$ (with respect to Lebesgue measure, which is the Haar measure for this group). Therefore we can form

$$
\left\langle f_{0}, \int_{0}^{\infty} h_{\rho}(z, t) \psi(\rho) d \rho\right\rangle
$$

as a kind of Fourier transform of $f_{0}$, with the mapping that assigns (4.8) to $\psi$ as a distribution on $(0, \infty)$. Here we keep $\alpha, \beta, \varepsilon$ fixed.

The first step of the proof, analogous to the proof of Lemma 2.1, is to show that these distributions have support at $\rho=1$. Now if $\psi$ is supported in $(1, \infty)$ then

$$
\left\langle f_{0}, \int h_{\rho} \psi(\rho) d \rho\right\rangle=\left\langle f_{0}, \mathscr{L}^{k} \int h_{\rho} \frac{\psi(\rho)}{(-\rho)^{k}} d \rho\right\rangle=\left\langle f_{k}, \int h_{\rho} \frac{\psi(\rho)}{(-\rho)^{k}} d \rho\right\rangle
$$

and so

$$
\left|\left\langle f_{0}, \int h_{\rho} \psi(\rho) d \rho\right\rangle\right| \leq M\left\|\int h_{\rho} \frac{\psi(\rho)}{(-\rho)^{k}} d \rho\right\|_{1} .
$$


We need to show that the right side of (4.9) tends to zero as $k \rightarrow \infty$, but this follows from the same integration by parts argument that shows that $\int h_{\rho} \psi(\rho) d \rho$ is integrable. Thus $\left\langle f_{0}, \int h_{\rho} \psi(\rho) d \rho\right\rangle=0$. Similarly, if $\psi$ is supported in $(0,1)$ then

$\left\langle f_{0}, \int h_{\rho} \psi(\rho) d \rho\right\rangle=\left\langle\mathscr{L}^{k} f_{-k}, \int h_{\rho} \psi(\rho) d \rho\right\rangle=\left\langle f_{-k}, \int h_{\rho}(-\rho)^{k} \psi(\rho) d \rho\right\rangle$

and $\left\|\int h_{\rho}(-\rho)^{k} \psi(\rho) d \rho\right\|_{1} \rightarrow 0$ as $k \rightarrow \infty$.

From the structure theorem for distributions with point support we conclude

$$
\left\langle f_{0}, \int h_{\rho} \psi(\rho) d \rho\right\rangle=\sum_{j=0}^{N} c_{j} \psi^{(j)}(1)
$$

for some set of constants $c_{j}$. But then

$$
\begin{aligned}
\left\langle f_{k}, \int h_{\rho} \psi(\rho) d \rho\right\rangle & =\left\langle f_{0}, \int h_{\rho}(-\rho)^{k} \psi(\rho) d \rho\right\rangle \\
& =\left.\sum_{j=0}^{N} c_{j}\left(\frac{\partial}{\partial \rho}\right)^{j}(-\rho)^{k} \psi(\rho)\right|_{\rho=1} .
\end{aligned}
$$

Since (4.10) is clearly a polynomial of degree $N$ in $k$ from the last expression and bounded in $k$ by (4.5) and the first expression, we conclude that $N=0$, hence

$$
\left\langle f_{0}, \int h_{\rho} \psi(\rho) d \rho\right\rangle=c \psi(1) .
$$

In (4.11) we have completed the analogue of the proof of Theorem 1.1, but it does not complete the proof of the present theorem. What is shown is

$$
\left\langle f_{0}+f_{1}, \int h_{\rho} \psi(\rho) d \rho\right\rangle=0
$$

since $f_{1}=\mathscr{L} f_{0}$ and $-\left.\rho \psi(\rho)\right|_{\rho=1}=-\psi(1)$. We need to show $f_{0}+f_{1}=0$. The problem is that we have required $\psi(\rho)$ to vanish near $\rho=0$. This causes a difficulty which can be seen if we observe that $\psi(0)=0$ implies that $\int h_{\rho} \psi(\rho) d \rho$ has total integral zero on $\mathrm{Heis}_{n}$, so that constants are automatically orthogonal to $\int h_{\rho} \psi(\rho) d \rho$. Thus at the very best (4.12) could only prove that $f_{0}+f_{1}$ is constant. In fact what we show in the next step of the proof is that $f_{0}+f_{1}$ is a function of $z$ alone, independent of $t$. For simplicity of notation we write $f=f_{0}+f_{1}$ and $g=\int h_{\rho} \psi(\rho) d \rho$.

We now exploit the group invariance of the hypotheses to conclude that (4.12) holds also for group translates of $f$, or in other words

$$
f * g=0
$$

for the group convolution on Heis $_{n}$. In order to show that $f$ is independent of $t$, or $T f=0$, we take a partial Fourier transform in the $t$-variable alone to transform the Heis ${ }_{n}$ convolution to a twisted convolution on $\mathbb{C}^{n}$. If we denote this partial Fourier transform by $\hat{f}(z, \tau)$ and $\hat{g}(z, \tau)$ then $(4.13)$ becomes

$$
\iint \hat{f}(z-w, \tau) \hat{g}(w, \tau) e^{i t \tau} e^{i(\tau / 2) \operatorname{Im} z \cdot \bar{w}} d w d \tau=0
$$


and in view of (4.6) and the form of $g$ this becomes

$$
\begin{aligned}
\iint \hat{f} & \left(z-w, \frac{\varepsilon \rho}{n+2|\alpha|}\right) \varphi_{\alpha, \beta, \varepsilon}\left(\frac{\sqrt{\rho} w}{\sqrt{4|\alpha|+2 n}}\right) \\
& \times \exp \left(\frac{\varepsilon i \rho}{2 n+4|\alpha|} \operatorname{Im} z \cdot \bar{w}\right) \psi\left(\frac{\rho}{n+2|\alpha|}\right) d \rho d w=0 .
\end{aligned}
$$

Of course $\hat{f}$ is only a tempered distribution, so the integrals in (4.15) must be understood in the distribution sense (of course

$$
\varphi_{\alpha, \beta, \varepsilon}\left(\frac{\sqrt{\rho} w}{\sqrt{4|\alpha|+2 n}}\right) \exp \left(\frac{\varepsilon i \rho}{2 n+4|\alpha|} \operatorname{Im} z \cdot \bar{w}\right) \psi\left(\frac{\rho}{n+2|\alpha|}\right)
$$

is a Schwartz class test function of $w$ and $\rho$, because $\psi$ vanishes in a neighborhood of zero). We can interpret (4.15) as saying

$$
\int \hat{f}(z-w, \tau) \varphi_{\alpha, \beta, \varepsilon}\left(\frac{\tau w}{\sqrt{2}}\right) e^{(i \tau / 2) \operatorname{Im} z \cdot \bar{w}} d w=0
$$

in the distribution sense for $\tau \neq 0$ and $\varepsilon=\operatorname{sgn} \tau$.

Now since $\varphi_{\alpha, \beta, \varepsilon}(z)$ forms an orthonormal basis of $L^{2}(\mathbb{C})$ for $\varepsilon$ fixed, we conclude that

$$
\int \hat{f}(z-w, \tau) g(w) e^{i(\tau / 2) \operatorname{Im} z \cdot \bar{u}} d w=0
$$

for $\tau \neq 0$ and any $L^{2}$ function $g$. This is a twisted convolution, and since $g$ is arbitrary it is easy to derive

$$
\hat{f}(z, \tau)=0 \text { for } \tau \neq 0
$$

by letting $g$ vary through an approximate identity.

Inverting the Fourier transform in (4.18) we obtain that $f(z, t)$ is a polynomial in $t$, and since $f$ is bounded we conclude that $f$ is independent of $t$. Recall that $f=f_{0}+f_{1}=f_{0}+\mathscr{L} f_{0}$, but the same argument could be applied to $f_{k}+\mathscr{L} f_{k}$. Thus we have

$$
f_{k}+\mathscr{L} f_{k}=g_{k}
$$

where $g_{k}(z, t)=g_{k}(z)$ is independent of $t$.

Now if we apply $\mathscr{L}$ to both sides of (4.19) and use (4.4) we obtain

$$
\mathscr{L} g_{k}=g_{k+1}
$$

and from (4.5) we obtain

$$
\left\|g_{k}\right\|_{\infty} \leq 2 M
$$

But since $g_{k}(z)$ is independent of $t$, we have $\mathscr{L} g_{k}=\Delta_{z} g_{k}$ where $\Delta_{k}$ denotes the Laplacian on $\mathbb{C}^{n}$ identified with $\mathbb{R}^{2 n}$. We can thus apply Theorem 1.1 to the sequence $\left\{g_{k}\right\}$ to conclude $g_{0}+g_{1}=0$. Combined with (4.19) this implies

$$
f_{k}+2 f_{k+1}+f_{k+2}=0 \text {. }
$$

But the recursion relation (4.22) is easily solved to yield

$$
f_{k}=(-1)^{k} f_{0}-k(-1)^{k}\left(f_{0}+f_{1}\right) \text {. }
$$


The uniform boundedness of $f_{k}$ then implies $f_{0}+f_{1}=0$, completing the proof.

Remark. The last step of the proof, combined with an induction argument, shows the following: if $\left\|L^{k} f\right\| \leq M$ for all $k \geq 0$ and $(L+1)^{j} f=0$ for some $j \geq 1$, then $(L+1) f=0$, where $L$ is any unbounded linear operator on a Banach space.

The same method of proof can be used to prove a version of Liouville's Theorem on the Heisenberg group, to the effect that if $f$ is a function on Heis ${ }_{n}$ of polynomial growth such that $\mathscr{L} f$ is a polynomial, then $f$ is a polynomial. This result is a special case of more general results of Geller [Ge 2], so we will not present the proof here.

\section{REFERENCES}

[Fo] G. Follard, Harmonic analysis in phase space, Ann. of Math. Stud., no. 122, Princeton Univ. Press, Princeton, N. J., 1989.

[Ga 1] J.-P. Gabardo, Tempered distributions with spectral gaps, Math. Proc. Cambridge Philos. Soc. 106 (1989), 143-162.

[Ga 2] __ Tempered distributions supported on a half-space of $\mathbb{R}^{n}$ and their Fourier transforms, Canad. J. Math. 43 (1991), 61-88.

[Ge 1] D. Geller, Fourier analysis on the Heisenberg group. I: Schwartz space, J. Funct. Anal. 36 (1980), 205-254.

[Ge 2] __ Liouville's theorem for homogeneous groups, Comm. Partial Differential Equations 8 (1983), 1665-1677.

[GS] I. M. Gelfand and G. E. Shilov, Generalized functions, vol. I, Academic Press, New York, 1964.

[He] S. Helgason, Groups and geometric analysis, Academic Press, New York, 1984.

[Ho] R. Howard, A note on Roe's characterization of the sine function, Proc. Amer. Math. Soc. 105 (1989), 658-663.

[HR] R. Howard and M. Reese, Characterization of eigenfunctions by boundedness conditions, Canad. Math. Bull. 35 (1992), 204-213.

[R] J. Roe, $A$ characterization of the sine function, Math. Proc. Cambridge Philos. Soc. 87 (1980), 69-73.

[RS] M. Reed and B. Simon, Methods of mathematical physics. Vol. II. Functional analysis, Academic Press, New York, 1980.

[SW] E. M. Stein and G. Weiss, Introduction to Fourier analysis on Euclidean spaces, Princeton Univ. Press, Princeton, N. J., 1971.

[Str 1] R. Strichartz, Harmonic analysis as spectral theory of Laplacians, J. Funct. Anal. 87 (1989), 51-148; Corrigendum, 109 (1992), 457-460.

[Str 2],$- L^{p}$ harmonic analysis and Radon transforms on the Heisenberg group, J. Funct. Anal. 96 (1991), 350-406.

Department of Mathematics, Cornell University, Ithaca, New York 14853 